\title{
Ultra-wide-band accumulation of coherent undulator synchrotron radiation in a resonating cavity
}

\author{
Y.H. Seo* \\ Department of Electrophysics, Kwangwoon University, Seoul 139-701, Korea \\ C. $\operatorname{Kim}^{\dagger}$ \\ School of General Studies, Gwangju Institute of Science and Technology, Gwangju 500-712, Korea
}

(Received 26 March 2011; published 21 June 2011)

\begin{abstract}
Cavity accumulation of coherent undulator synchrotron radiation emitted by a train of periodic electron bunches is investigated. Phase-matching conditions for accumulation of radiation emitted by successive bunches are analyzed and numerically confirmed. While the coherent emission of a single bunch is optimal at grazing resonance, the accumulated radiation targeted at the upper resonant frequency of the waveguide mode is found to have much broader bandwidth and higher efficiency as the resonance steps away from the grazing condition. Numerical results confirm that stimulated superradiance is responsible for the accumulated radiation.
\end{abstract}

DOI: 10.1103/PhysRevSTAB.14.060706

PACS numbers: 41.60.Cr, 52.59.-f, 42.72.Ai

\section{INTRODUCTION}

Coherent effect $[1,2]$ or superradiance describes emission of radiation from a group of radiators whose individual fields are emitted in phase so that they may add up constructively. While the power of usual incoherent radiation is proportional to the number of radiators, that of superradiant emission is proportional to its square. Coherent emission of relativistic electrons bunched within a length shorter than the wavelength of emitted radiation is an example. Recently, Carr et al. [3] demonstrated that the coherent effect in a subpicosecond electron bunch enhanced the power level of synchrotron radiation emission in the terahertz $(\mathrm{THz})$ frequency range [4] by many orders of magnitude. Similar enhancement of radiation emission has been observed in various other emission processes such as transition radiation $[5,6]$, undulator radiation $[7,8]$, and Cherenkov radiation [9].

Rather than the coherent emission of a single bunch, the present work considers cumulative effect of repeated bunches in a resonating cavity. Periodic microbunches delivered from compact rf accelerators [10,11] can be an example of such repeated bunches. The bunch interval is typically in a nanosecond range. The beneficial effect that is expected from repeated bunches is the stimulated superradiance [12-15]. In other words, the radiation field excited by earlier bunches may stimulate radiation emission of the following bunches, enhancing their individual

*yseo@kw.ac.kr
† ckim@gist.ac.kr

Published by the American Physical Society under the terms of the Creative Commons Attribution 3.0 License. Further distribution of this work must maintain attribution to the author(s) and the published article's title, journal citation, and DOI. superradiance. The purpose of this paper is to describe the required condition for it.

We imagine a rectangular cavity of length $L$ immersed in a plane-polarized undulator field of period $\lambda_{w}=2 \pi / k_{w}$. Assuming that a single eigenmode in the transverse direction is excited in the cavity, we write the vector potential of the radiation field as a sum of the axial Fourier components:

$$
\vec{A}_{s}=\frac{\hat{x}}{2} E(x, y) \sum_{n=1}^{\infty} A_{n}(t) \sin \left(k_{n} z\right) e^{-i \omega_{n} t}+\text { c.c. }
$$

where $\hat{x}$ is the polarization unit vector, $E(x, y)$ is the transverse eigenfunction of the excited modes, $A_{n}(t), k_{n}=$ $n \pi / L$, and $\omega_{n}=c \sqrt{k_{n}^{2}+k_{\perp}^{2}}$ are the amplitude, the axial wave number, and the frequency of the $n$th mode, respectively, $k_{\perp}$ is the transverse wave number, and c.c. stands for the complex conjugate.

Temporal evolution of each mode follows the wellknown free-electron laser dynamics [16]. The amplitude of the $n$th mode is governed by [17]

$$
\frac{d}{d t} A_{n}(t)=\frac{C_{E}}{\omega_{n}}\left\langle\frac{1}{\gamma_{j}} e^{i \psi_{n}\left(t, z_{j}\right)}\right\rangle
$$

where $\langle\cdots\rangle$ denotes the average over all electrons in the cavity, $\gamma_{j}$ and $z_{j}$ are the normalized energy and axial coordinate of the $j$ th electron, respectively, and $\psi_{n}\left(t, z_{j}\right)=$ $\omega_{n} t-\left(k_{n}+k_{w}\right) z_{j}$ is the phase of electron oscillation relative to the $n$th mode. Moreover, $C_{E}$ is the coupling constant given by

$$
C_{E}=2 S_{E} A_{w} \omega_{p}^{2}\left(V_{b} / V_{C}\right),
$$

where $A_{w}$ is the undulator amplitude, $\omega_{p}^{2}=4 \pi n_{0} e^{2} / m_{0}$ is the squared electron plasma frequency, $n_{0}$ is the average 
electron density within the bunch, $V_{b}$ and $V_{C}$ are the volumes of the bunch and the cavity, respectively, and $S_{E}$ is the average value of $E(x, y)$ over the cavity cross section. For $\mathrm{TE}_{01}$ mode, $S_{E}=1 / 2$.

\section{CONDITIONS FOR RADIATION ACCUMULATION}

Let us now consider the $m$ th bunch which enters the cavity at time

$$
t_{m}=m T_{b} \quad(m=0,1,2, \ldots),
$$

where $T_{b}$ is the bunch period. The average axial position of each electron in the $m$ th bunch at time $t$ may be written as

$$
z_{j}=c \bar{\beta}_{z}\left(t-t_{m}\right)+\zeta_{j},
$$

where $c \bar{\beta}_{z}$ is the axial speed averaged over an undulator period, and $\zeta_{j}$ is the position relative to the bunch center. In the linear regime, temporal change of $\zeta_{j}$ during travel may be neglected. Superradiant emission occurs when $\zeta$ 's distribute nonuniformly within a wavelength of excited radiation. Inserting Eq. (5), we can rearrange the phase $\psi_{n}\left(t, z_{j}\right)$ as

$$
\psi_{n}\left(t, z_{j}\right)=\omega_{n} t_{m}+\Delta \omega_{n}\left(t-t_{m}\right)-\left(k_{n}+k_{w}\right) \zeta_{j}
$$

where $\Delta \omega_{n} \equiv \omega_{n}-\left(k_{n}+k_{w}\right) c \bar{\beta}_{z}$ is the detuning parameter. When Eq. (2) is integrated for the phase of Eq. (6), we obtain the increment of mode amplitude produced by the $m$ th bunch during $t_{m}<t<t_{m}+L / c \bar{\beta}_{z}$ :

$$
\Delta A_{n} \simeq \frac{C_{E}}{\omega_{n} \gamma_{0}} g_{m}\left(k_{n}\right) e^{i \omega_{n} t_{m}} \int_{0}^{L / c \bar{\beta}_{z}} e^{i \Delta \omega_{n} t^{\prime}} d t^{\prime},
$$

where $g_{m}\left(k_{n}\right)=\left\langle\exp \left[-i\left(k_{n}+k_{w}\right) \zeta_{j}\right]\right\rangle$ works as the form factor that measures the coherent effect in the $m$ th bunch. The integral in Eq. (7) is peaked at resonance points where the detuning parameter vanishes. The resonant wave numbers that satisfy $\Delta \omega_{n}=0$ are

$$
k_{ \pm}=\left(\gamma_{z} \bar{\beta}_{z}\right)^{2} k_{w}\left[1 \pm \frac{s}{\bar{\beta}_{z}}\right]
$$

where $\gamma_{z}=1 / \sqrt{1-\bar{\beta}_{z}^{2}}$ and

$$
s=\sqrt{1-\left(\frac{k_{\perp} / k_{w}}{\gamma_{z} \bar{\beta}_{z}}\right)^{2}} .
$$

The superradiant emission of each bunch will contain a broad spectrum of axial modes around these resonant modes. For a cavity of length $L$, its bandwidth is scaled by $\delta k / k_{ \pm} \sim \lambda_{w} / L$. However, our interest is not in this individual superradiance, but in its long-term accumulation.

As seen in Eq. (7), the cavity modes emitted by each bunch have a bunch-dependent phase factor, $\exp \left(i \omega_{n} t_{m}\right)$. For constructive accumulation over successive bunches, this phase factor should be ignorable, i.e.,

$$
\omega_{n} T_{b}=2 \pi \times \text { integer. }
$$

While the modes that satisfy Eq. (10) can accumulate their growth provided by each bunch, those that do not will experience oscillation over successive bunches with no significant growth.

Targeting constructive accumulation of the modes near the upper resonance frequency, $\omega_{+}=c\left(k_{+}^{2}+k_{\perp}^{2}\right)^{1 / 2}$, we expand the mode frequency in powers of $\delta k_{n}\left(=k_{n}-k_{+}\right)$:

$$
\omega_{n}=\omega_{+}+c \beta_{g}^{+} \delta k_{n}+\frac{c^{4} k_{\perp}^{2}}{2 \omega_{+}^{3}}\left(\delta k_{n}\right)^{2} .
$$

Here, $c \beta_{g}^{+}=k_{+} c^{2} / \omega_{+}$is the group velocity at $k_{n}=k_{+}$. Broadband accumulation is expected when the zeroth and the first order frequencies satisfy the accumulation condition [Eq. (10)] separately.

Substituting $\delta k_{n}=\delta n(\pi / L)$, where $\delta n$ is an integer, we obtain the condition for the first order frequency as $T_{b}\left(c \beta_{g}^{+} / 2 L\right) \delta n=$ integer. This condition may also be written as

$$
T_{b}=f_{1} T_{R}, \quad\left(f_{1}=1,2, \ldots\right),
$$

where $T_{R}=2 L / c \beta_{g}^{+}$is the round-trip time along the cavity at the group velocity, and $f_{1}$ may be assumed as an integer without loss of generality [18]. Equation (12) can be interpreted as a synchronization condition. When it is satisfied, bunches enter into the cavity in every $f_{1}$ circulation time of the radiation pulse.

The condition for the zeroth order (resonant mode) frequency, combined with the synchronization condition, reads $\left(\omega_{+} / 2 \pi\right) f_{1}\left(2 L / c \beta_{g}^{+}\right)=$integer. Inserting here $\omega_{+}=c \bar{\beta}_{z}\left(k_{w}+k_{+}\right)$and $k_{+}=n_{+} \pi / L$, we may rewrite it as

$$
\left(2 N_{w}+n_{+}\right) f_{1}(1-\varepsilon)=\text { integer, }
$$

where $n_{+}$is the mode number at upper resonance, $N_{w}=$ $L / \lambda_{w}$ is the cavity length in units of the undulator period, and $\varepsilon=1-\bar{\beta}_{z} / \beta_{g}^{+}$is the slippage parameter for the upper resonant mode.

The range of accumulated modes is determined by the second order frequency, $\omega_{n}^{(2)}$, which destroys phase matching up to the first order. Suppose that the slight phase mismatch introduced by $\omega_{n}^{(2)}$ accumulates to reach $2 \pi$ by the time the $M$ th bunch enters, i.e., $\omega_{n}^{(2)} t_{M}=2 \pi$. Then, the mode amplitude accumulated up to that bunch will be null, simply because the relative phases of the wavelets excited by $M$ bunches distribute uniformly from zero to $2 \pi$. This argument predicts that the excitation bandwidth at time $t$ should be

$$
\Delta k \simeq \sqrt{\frac{4 \pi \omega_{+}^{3}}{c^{4} k_{\perp}^{2} t}} .
$$


Corresponding spatial width of the radiation pulse, in units of the resonant wavelength, amounts $\Delta x / \lambda_{+} \sim$ $c \sqrt{\left(k_{\perp}^{2} / 4 \pi \omega_{+}\right) t}$. Hence, the pulse width disperses in proportion to $\sqrt{t}$.

The accumulation condition, Eq. (13), can be fulfilled in a number of ways. The simplest case may be the one in which $\varepsilon=0$ and $f_{1}=1$. Zero slippage $(\varepsilon=0)$ occurs at the grazing resonance (Fig. 1) where the two resonant wave numbers of Eq. (8) overlap by $s=0$, i.e.,

$$
k_{\perp}=\gamma_{z} \bar{\beta}_{z} k_{w} .
$$

Superradiance of an electron bunch at grazing resonance has received some attention in a waveguide free-electron laser. The zero-slippage condition allows continual energy transfer from the electron bunch to the radiation pulse. The pulse width is limited only by dispersion, resulting in ultrawide-band excitation $[19,20]$. In the resonating cavity, coherent excitation of individual bunches can be accumulated over a broadband if we assume Eq. (13), which reduces in the grazing resonance case to

$$
2 N_{w}=\text { integer. }
$$

In other words, accumulation of near-grazing modes over successive bunches is achieved when the cavity length is an integer or a half-integer multiple of the undulator period.

Zero slippage is a crucial condition for broadband excitation in a long waveguide. However, in a cavity of short length, broadband accumulation is not limited to the zeroslippage grazing case. At the group velocity of the upper resonant frequency, the slippage length $\ell_{s}$ in units of the resonant wavelength $\lambda_{+}$is estimated to be $\ell_{s} / \lambda_{+}=$ $N_{w} \beta_{g}^{+} s$. Therefore, if the cavity length is confined within just a few undulator periods, the effect of slippage should not be a critical issue. Then, the advantage of stepping

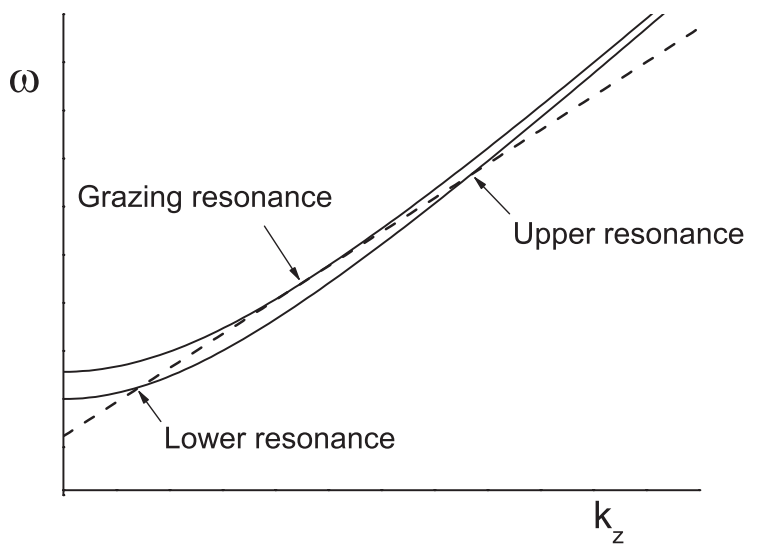

FIG. 1. Dispersion curves of the beam mode [broken line; $\omega=$ $\left.\left(k_{z}+k_{w}\right) c \bar{\beta}_{z}\right]$ and the waveguide mode (solid lines; $\omega=c \sqrt{k_{z}^{2}+k_{\perp}^{2}}$ ). While two curves intersect at two points for $k_{\perp}<\gamma_{z} \bar{\beta}_{z} k_{w}$, the grazing resonance where two curves meet tangentially occurs when $k_{\perp}=\gamma_{z} \bar{\beta}_{z} k_{w}$. away from grazing resonance is apparent. The emission frequency (at upper resonance) can be much higher than the frequency of the grazing mode, and at the same time, as Eq. (14) predicts, radiation accumulation can proceed over a much broader bandwidth.

One way to fulfill Eq. (13) for accumulation at an offgrazing resonance and its neighborhood is to assume $2 N_{w}=$ integer and

$$
\left(2 N_{w}+n_{+}\right) f_{1} \varepsilon=\text { integer. }
$$

Substituting $2 N_{w}+n_{+}=2 N_{w}\left(1+k_{+} / k_{w}\right)=2 N_{w} \gamma_{z}^{2}(1+$ $\left.\bar{\beta}_{z} s\right)$ and $\varepsilon=\left(1 / \gamma_{z}^{2}\right)\left[s /\left(\bar{\beta}_{z}+s\right)\right]$, we find that the requirement is equivalent to

$$
2 N_{w} f_{1} \frac{s\left(1+\bar{\beta}_{z} s\right)}{\bar{\beta}_{z}+s}=f_{2} \quad\left(f_{2}=1,2, \ldots\right) .
$$

Once $f_{1}$ and $f_{2}$ are chosen, Eq. (18) for $s$ defines the transverse dimension of the cavity. An approximate relation at relativistic electron energies $\left(\bar{\beta}_{z} \approx 1\right), s \simeq$ $f_{2} /\left(2 N_{w} f_{1}\right)$, suggests that several choices are available for $f_{1}$ and $f_{2}$ in $0<s<1$. The case that brings the highest resonant frequency will be the one with $f_{2}=2 N_{w} f_{1}-1$.

\section{NUMERICAL RESULTS}

In order to confirm the investigated conditions for radiation buildup, a numerical code has been written. The code injects electron bunches periodically into a cavity, and then calculates evolution of the cavity modes by integrating Eq. (2) along with the equations of motion for the bunch electrons. Each bunch is consisted of a few hundred particles typically. Here we present the results of the following three cases: (A) the grazing case with $f_{1}=1$ and $f_{2}=0$; (B) an off-grazing case with $f_{1}=2$ and $f_{2}=4$; (C) another off-grazing case with $f_{1}=1$ and $f_{2}=3$. Parameter values used in simulation have been summarized in Table I. If $\lambda_{w}=10 \mathrm{~cm}$, as an example, the actual resonance frequencies of the three cases are 0.30, 0.45, and $0.53 \mathrm{THz}$, respectively. Each bunch contains 17. pC charge in 0.53 ps duration. The bunch injection frequencies are 754, 376, and 750.5 MHz, respectively.

TABLE I. Input parameters used in simulation.

\begin{tabular}{lcc}
\hline \hline Physical quantity & Symbol $^{\mathrm{a}}$ & Value \\
\hline Undulator strength & $e A_{w} / m c^{2}$ & 1 \\
Electron energy & $\gamma_{0}$ & 12.31 \\
Doppler parameter & $\gamma_{z} \bar{\beta}_{z}$ & 10 \\
Bunch length & $k_{w} \ell_{b}$ & 0.01 \\
Coupling constant & $\left(e / m c^{4} k_{w}^{2}\right) C_{E}$ & $10^{-4}$ \\
Cavity length & $N_{w}\left(=L / \lambda_{w}\right)$ & 2 \\
Cavity cross section & $k_{w}^{2} a b$ & $\pi^{2} / 50$ \\
Cavity height $(\mathrm{A} / \mathrm{B} / \mathrm{C})$ & $k_{w} b$ & $0.314 / 0.362 / 0.475$ \\
Exited cavity mode & $\mathrm{TE}_{01 n}$ & \\
\hline \hline
\end{tabular}

${ }^{\mathrm{a}}$ Normalized quantities used in simulation. 
Figure 2 shows the spectral distribution of the mode amplitude in the three cases after 20 bunches have been injected. The accumulation bandwidths are in good agreement with Eq. (14). Figure 2(d) shows the actual pulse shape of the case $\mathrm{C}$, formed by over 400 modes within the excitation band $(\Delta n= \pm 220)$. Resulting peak amplitude is 2 orders of magnitude greater than the amplitudes of the individual modes.

The observed bandwidth ratio for the three cases is $A: B: C \simeq 1: 1.5: 3.5$. Consequences of this bandwidth difference appear in the peak power and duration of the radiation pulse. Figure 3(a) compares the radiation power of the three cases as a function of the bunch number. It is seen that the power ratio of the three cases is approximately equal to the square of the bandwidth ratio.

The power plotted in Fig. 3(a) is the Poynting vector integrated and averaged over the cavity cross section that follows the bunches during their flight. Hence, it is the average background radiation power felt by bunch electrons during their flight in the cavity. We argue that this background radiation is stimulating the radiation emission in successive periodic bunches. A convenient indicator for the emission rate will be the energy loss of electrons during their flight. Figure 3(b) shows the relation between the electron energy loss and the background radiation power. The log-log plots for the three cases distribute approximately on a line of slope $1 / 2$, implying that the emission rate is proportional to the square root of the background radiation power. In fact, in the limit of zero bunch length, the equation of motion for the bunch electrons estimates the efficiency as

$$
\eta \simeq \pi N_{w} a_{0} \frac{\bar{\beta}_{z}}{\gamma_{0}\left(\gamma_{0}-1\right)} \sqrt{\frac{P[M W]}{347.5 S}}
$$

where $S=k_{w}^{2} a b$ is the normalized cross-sectional area of the cavity. For the assumed parameter values in simulation, Eq. (19) corresponds to the broken line added in Fig. 3(b). The square root dependence on power is a well-known property of the stimulated superradiance $[12,13]$. From Fig. 3(b), we conclude that stimulated superradiant emission is responsible for the radiation accumulated over periodic bunches.

Several isolated peaks away from the resonant region are seen in the spectral distributions shown in Fig. 2. Although the total power contained in those modes is relatively
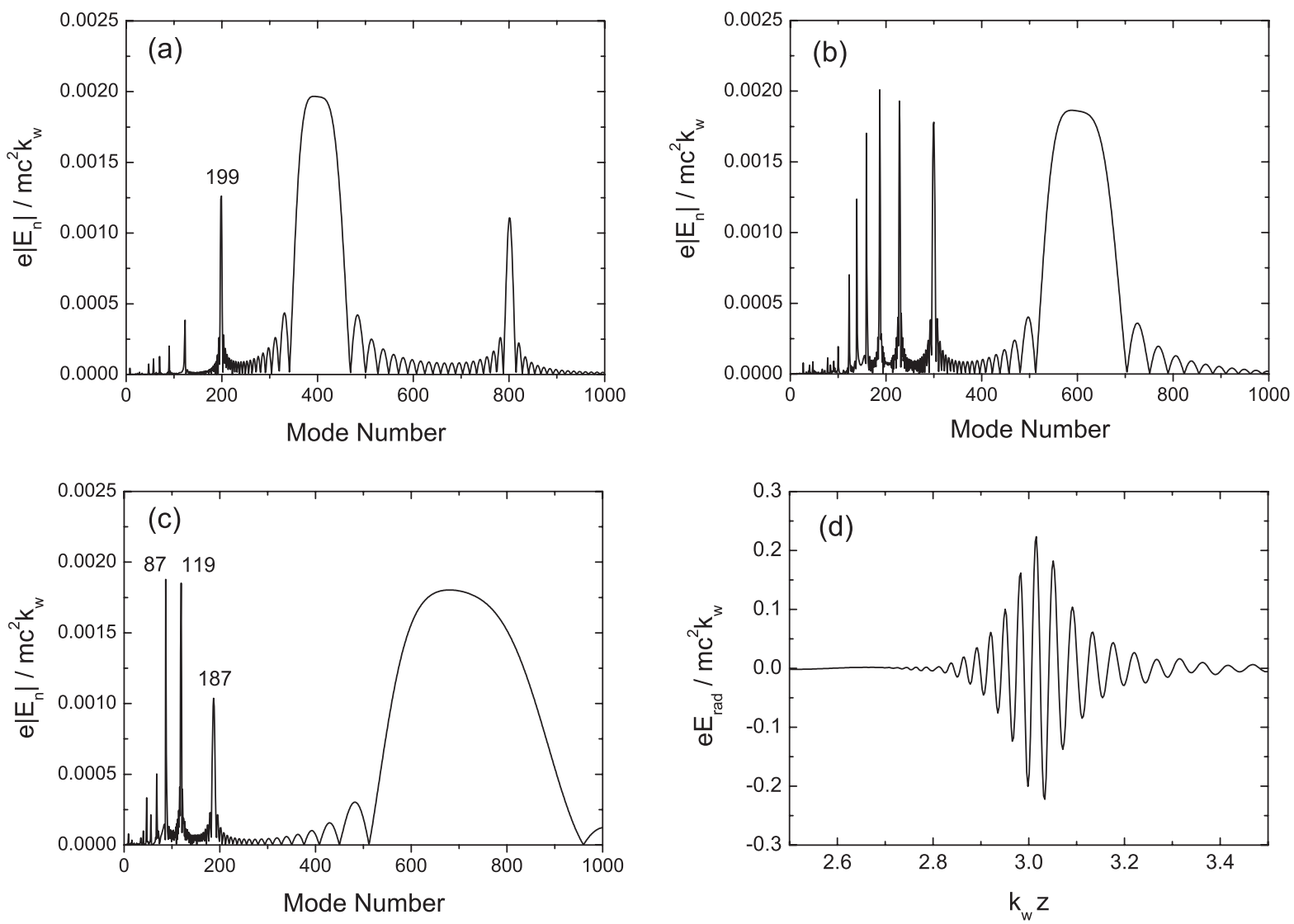

FIG. 2. Spectral distribution of the mode amplitude accumulated by the first 20 bunches in three simulated cases. $e\left|E_{n}\right| / m c^{2} k_{w}$ is the normalized field $\left(E_{n}=\omega_{n} A_{n} / c\right)$ of the $n$th mode. (a) Case A where $T_{b}=T_{R}$ and $k_{\perp}=10 k_{w}$ (a grazing resonance case). (b) Case B where $T_{b}=2 T_{R}$ and $k_{\perp}=8.86 k_{w}$. (c) Case C where $T_{b}=T_{R}$ and $k_{\perp}=6.620 k_{w}$. (d) Actual pulse shape of radiation field produced from the spectral distribution of (c). 

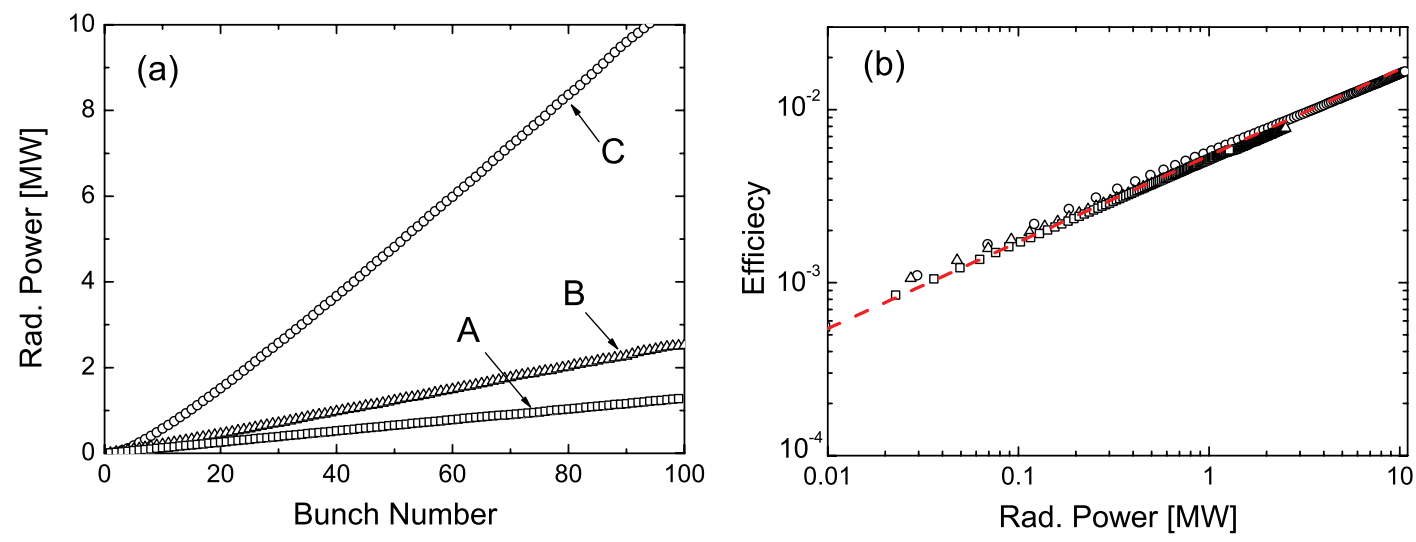

FIG. 3. Power and efficiency evolution for cases A (squares), B (triangles), and C (circles). (a) Average radiation power measured at each bunch. (b) Average energy loss of each bunch as a function of the radiation power. The broken line (in red) is an analytic estimation [Eq. (19)] for a pointlike bunch.

insignificant, it will be worthwhile to explain how they are excited. The fact that these modes grow over a long period of time implies that they are also satisfying the accumulation condition. When Eqs. (12) and (13) are assumed for the resonant and the near-resonant modes to be accumulated, the accumulation condition [Eq. (10)] for an offresonant mode having a nonzero detuning parameter, $\Delta \omega_{n}$, becomes

$$
\Delta \omega_{n} f_{1} T_{R} / 2 \pi-\left(n+2 N_{w}\right) f_{1} \varepsilon=p,
$$

where $p$ is an integer. The wave numbers that satisfy Eq. (20) are

$$
k_{n}=\left(\gamma_{g} \beta_{g}^{+}\right)^{2} k_{w}\left[\xi_{p} \pm \frac{1}{\beta_{g}^{+}} \sqrt{\xi_{p}^{2}-\left(\frac{k_{\perp} / k_{w}}{\gamma_{g} \beta_{g}^{+}}\right)^{2}}\right],
$$

where $\gamma_{g}=1 / \sqrt{1-\beta_{g}^{+2}}$ and $\xi_{p}=1+p / 2 N_{w} f_{1}$. It can be immediately identified that the peaks in Fig. 2(c), 87,
119, and 187, correspond to $p=0,-1$, and -2 , respectively.

Let us finally mention that, due to their long-term nature, the accumulation conditions are rather sensitive to small variations in $k_{\perp}$ and $N_{w}$, i.e., in the cavity dimension. Figure 4 shows a result of small change in $k_{\perp}$ from the case C: $k_{\perp}=6.569$. It corresponds to $\sim 0.4 \%$ mismatch in the cavity height. The accumulated field shows no significant difference up to 20 bunches. However, as time goes by, small phase mismatch accumulates to develop a hollow structure in the mode spectrum as seen in Fig. 4(a). Resulting radiation pulse disperses to split [Fig. 4(b)]. Similar sensitivity to $N_{w}$, the cavity length, is also seen in Fig. 5.

\section{DISCUSSION AND CONCLUSION}

Numerical results shown in the previous section do not include the effect of finite cavity $Q$. For finite $Q$, we can imagine a steady state in which stimulated superradiance is

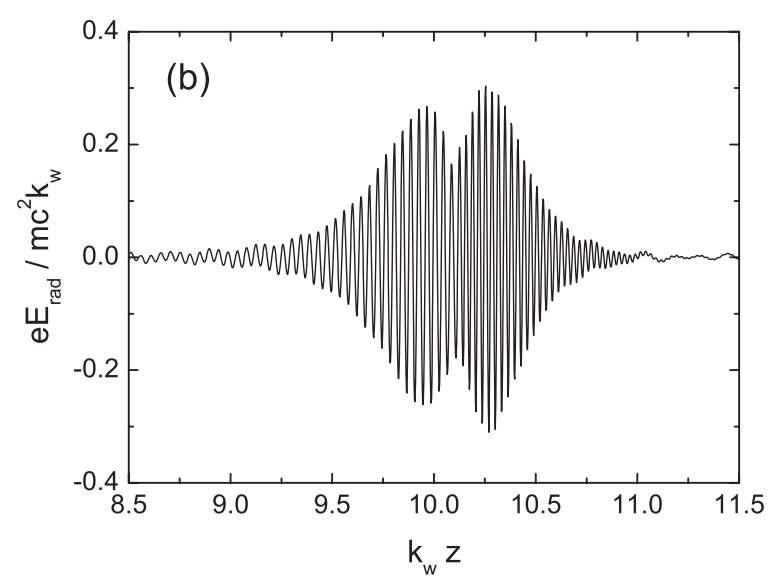

FIG. 4. Radiation accumulated by 100 bunches in case $\mathrm{C}$ with a small mismatch in $k_{\perp}: k_{\perp}=6.596 k_{w}$. (a) Mode amplitude spectrum. The broken line added for comparison is the exact case $\mathrm{C}\left(k_{\perp}=6.620 k_{w}\right)$ at the same moment. (b) The pulse shape of radiation field produced from (a). 

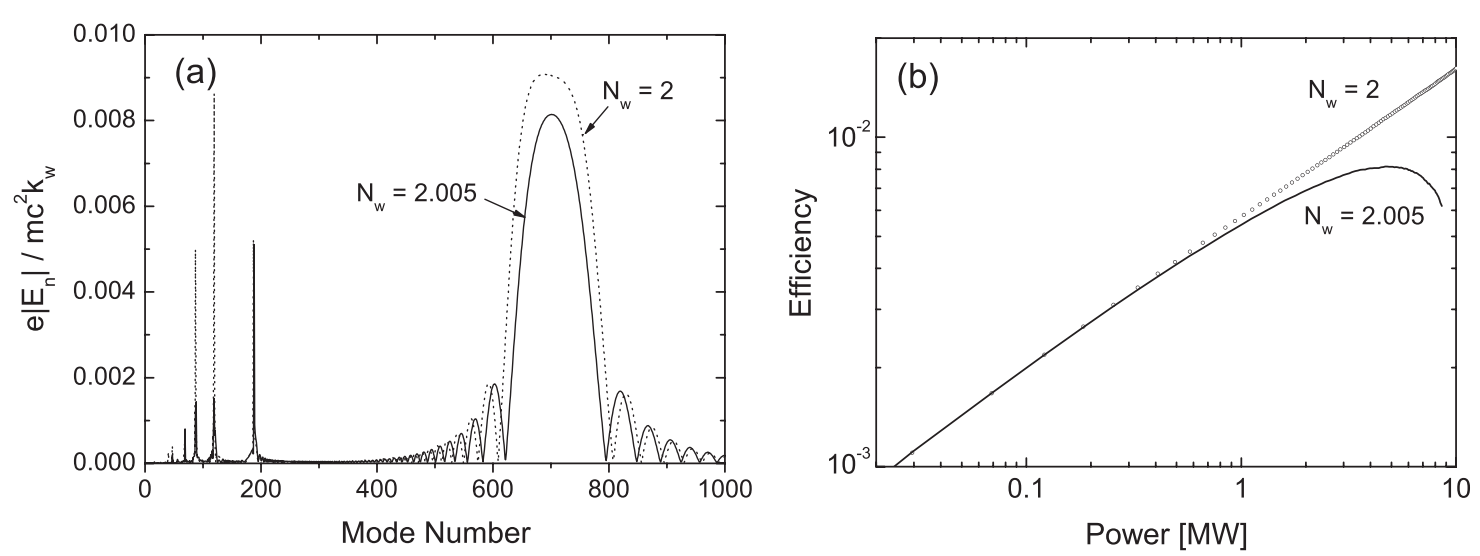

FIG. 5. Radiation accumulated by 100 bunches for case C with $N_{w}=2.005$ (a) Mode amplitude spectrum. The broken line shows the exact case $\mathrm{C}$ amplitude at the same time. (b) Efficiency comparison.

balanced with energy loss due to output coupling and wall resistance. In such a steady state, the system loses its memory beyond the characteristic decay time, $\tau_{d}$. In other words, the radiation pulses are formed by emission which occurred within the past time of order $\tau_{d}$. One of the consequences that it brings concerns the long-term sensitivity to the cavity dimension, seen in Figs. 4 and 5. Permitted error ranges in the cavity dimension can be determined in such a way that their significant effects do not appear within the decay time. Another consequence may be that the bandwidth of accumulation saturates and that the corresponding pulse width stops to disperse in space. The saturated bandwidth is given by Eq. (14) with $\tau_{d}$ at the place of the time $t$ :

$$
\Delta k \simeq \sqrt{\frac{4 \pi \omega_{+}^{3}}{c^{4} k_{\perp}^{2} \tau_{d}}} .
$$

The cavity works as a stable oscillator in the steady state, as long as periodic bunches are supplied. The saturated radiation pulse(s) will circulate in the cavity, emitting periodic bursts of high-power ultra-wide-banded $\mathrm{THz}$ waves through an output coupling.

In conclusion, ultra-wide-band accumulation of coherent undulator synchrotron radiation emitted by periodic electron bunches in a resonating cavity has been investigated. Phase-matching consideration over successive bunches shows that broadband accumulation is possible if the bunches are synchronized to the generated radiation pulse. Conditions for accumulation targeted at the upper resonant frequency have been obtained, and confirmed by numerical simulation. While the ultra-wide-band emission of a single bunch in a long waveguide is restricted around the grazing resonance, ultra-wide-band accumulation of superradiance in a short cavity is found to exhibit wider bandwidth and higher power at off-grazing resonances. The simulation results also reveal that the emission rate is proportional to the square root of the background radiation power, suggesting that the accumulated radiation is due to stimulated superradiance of periodic bunches. When cavity $Q$ is included, the cavity can work as a stable oscillator. The problem of sensitivity to the cavity dimension over a long period of time may not be an issue when the cavity $Q$ is finite.

\section{ACKNOWLEDGMENTS}

This work was supported by Kwangwoon University under the 2011 Internal Research Grant.

[1] Y. U. Jeong, Y. Kawamura, K. Toyoda, C. H. Nam, and S. S. Lee, Phys. Rev. Lett. 68, 1140 (1992).

[2] D. A. Jaroszynski, R. J. Bakker, A. F. G. van der Meer, D. Oepts, and P. W. van Amersfoort, Phys. Rev. Lett. 71, 3798 (1993).

[3] G. L. Carr, M. C. Martin, W. R. McKinney, K. Jordan, G. R. Neil, and G.P. Williams, Nature (London) 420, 153 (2002).

[4] M. Tonouchi, Nat. Photon. 1, 97 (2007).

[5] W. P. Leemans, C. G. R. Geddes, J. Faure, C. Toth, J. Van Tilborg, C. B. Schroeder, E. Esarey, G. Fubiani, D. Auerbach, B. Marcelis, M. A. Carnahan, R. A. Kaindl, J. Byrd, and M. Martin, Phys. Rev. Lett. 91, 074802 (2003).

[6] S. Casalbuoni, B. Schmidt, P. Schmüser, V. Arsov, and S. Wesch, Phys. Rev. ST Accel. Beams 12, 030705 (2009).

[7] A. Abramovich, Y. Pinhasi, V. Shterngartz, L. Gilutin, H. Kleinman, A. Eichenbaum, I. M. Yakover, M. Cohen, A. Gover, B. Levush, T. M. Antonsen, and V. L. Granatstein, Nucl. Instrum. Methods Phys. Res., Sect. A 375, 164 (1996).

[8] Y. Shibata, S. Sasaki, and K. Ishi, Nucl. Instrum. Methods Phys. Res., Sect. A 483, 440 (2002).

[9] A. M. Cook, R. Tikhoplav, S. Y. Tochitsky, G. Travish, O. B. Williams, and J.B. Rozenzweig, Phys. Rev. Lett. 103, 095003 (2009).

[10] Y. C. Huang, H. Wang, R. H. Pantell, J. F. Schmerge, J. W. Leweelen, and J. Feinstein, IEEE J. Quantum Electron. 31, 1637 (1995). 
[11] P. Kung, H.-C. Lihn, H. Wiedemann, and D. Bocek, Phys. Rev. Lett. 73, 967 (1994).

[12] I. Schnitzer and A. Gover, Nucl. Instrum. Methods Phys. Res., Sect. A 237, 124 (1985).

[13] M. Arbel, A. Abramovich, A. L. Eichenbaum, A. Gover, H. Kleinman, Y. Pinhasi, and I. M. Yakover, Phys. Rev. Lett. 86, 2561 (2001).

[14] A. Gover, E. Dyunin, Y. Lurie, Y. Pinhasi, and M. V. Krongauz, Phys. Rev. ST Accel. Beams 8, 030702 (2005).

[15] V. Zhaunerchyk, R. T. Jongma, Y. Lurie, Y. Pinhasi, and W. J. van der Zande, Appl. Phys. Lett. 97, 231109 (2010).

[16] C. A. Brau, Free-Electron Lasers (Academic, New York, 1990).

[17] Y.H. Seo and W.H. Lee, IEEE Trans. Plasma Sci. 38, 2016 (2010).
[18] In general, $f_{1}$ is a rational number. However, if it is not an integer, more than a single pulse is excited in the cavity. For example, if $f_{1}=1 / 2$, every other neighboring mode is accumulated to form two pulses in the cavity. Although bunch injection is twice as frequent as pulse round-trip, only half of the injected bunches interact with a given pulse. Hence, as far as interaction with a given pulse is concerned, we may take the effective bunch period as the round-trip time of that pulse, i.e., $T_{b}=T_{R}$. We can assume $f_{1}$ as an integer without loss of generality, in this sense.

[19] A. Gover, F. V. Hartemann, G. P. Le Sage, N. C. Luhmann, Jr., R. S. Zhang, and C. Pellegrini, Phys. Rev. Lett. 72, 1192 (1994).

[20] Y. Pinhasi, Yu. Lurie, and A. Yahalom, Phys. Rev. E 71, 036503 (2005). 